\title{
Inmunoterapia local
}

\section{Local immunotherapy}

\section{E. Lasa, S. Garrido, E. Arroabarren, M. Anda, B. Ollo, A.I. Tabar}

\section{RESUMEN}

La inmunoterapia específica, junto con la evitación del alergeno y el tratamiento sintomático, forma parte del tratamiento de la patología alérgica. La modalidad más antigua, más conocida y mejor estudiada es la inmunoterapia subcutánea (ITSC), cuya eficacia tanto a corto como a largo plazo, ha sido ampliamente demostrada en numerosos estudios. Sin embargo, a pesar de haberse demostrado segura, no está exenta de efectos adversos y precisa ser administrada bajo supervisión de personal médico. Esto ha animado a buscar nuevas vías de administración de eficacia similar, con un buen perfil de seguridad, y de buena cumplimentación por parte del paciente. De las distintas alternativas estudiadas la más relevante es la inmunoterapia sublingual (ITSL). En ésta, se administra el antígeno en forma de gotas debajo de la lengua. Existen diferentes pautas de administración en función del alergeno implicado. La dosis óptima de tratamiento está aún sin determinar, dosis optima de tratamiento está aún sin determinar, hallándose en este momento en un rango amplio de dosis respecto a la innunoterapia subcutanea. Su mecanismo de acción es poco conocido aunque en diversos estudios se han observado cambios inmunológicos. La ITSL ha mostrado un buen perfil de seguridad con escasos efectos secundarios, habitualmente de carácter local. Asimismo se han realizado distintos ensayos clínicos en los que se ha demostrado su eficacia en el tratamiento de la alergia respiratoria tanto en niños como en alultos. Por ello, aunque aún existen niños como en adultos. Por ello, aunque aún existen datos sin resolver respecto a esta via de administración de inmunoterapia, ha sido propuesta por la OMS como una alternativa válida a la ITSC.

Palabras clave. Inmunoterapia. Inmunoterapia sublingual. Inmunoterapia intranasal. Inmunoterapia local. Alergia respiratoria.

An. sis. sanit. Navar. 2003; 26 (Supl. 2): 111-118.

\section{ABSTRACT}

Specific immunotherapy, together with avoidance of the allergen and symptomatic treatment, forms part of the treatment of allergic pathology. The oldest, best known and most studied form is subcutaneous immunotherapy (SCIT), whose efficacy, both in the short and the long term, has been widely demonstrated in numerous studies. However, in spite of having been shown to be safe, it is not free of adverse effects and must be administered under the adverse effects and must be ad supervision of medical personnel. This has encourage the search for new ways of administration of simila efficacy, with a good safety profile and good adherence on the patient's side. Sublingual immunotherapy (SLIT) is the most relevant of the different alternative studied. In this alternative the antigen is administered in the form of drops under the tongue. There are different dosages of administration depending on the allergen involved. The optimum treatment dose has still to be determined, at present a wide range of still to be determined, at present a wide range of dosages are found in comparison with subcutaneous immunotherapy. Its mechanism of action is little known although immunological changes have been observed in different studies. SLIT has shown a good safety profile with scarce secondary effects, normally of a local character. Similarly, different clinical tests have been carried out in which its efficacy has been shown in the treatment of respiratory allergy both in children and in adults. For this reason, although there are still che still unresolved data concerning this way of administering as a valid alternative to SCIT.

Key words. Immunotherapy. Sublingual immunotherapy. Intranasal immunotherapy. Local immunotherapy. Respiratory allergy.
Sección de Alergología. Hospital Virgen del Camino. Pamplona.

\author{
Correspondencia: \\ Eva $\mathrm{M}^{\mathrm{a}}$ Lasa Luaces \\ Sección de Alergología \\ C. Salud Conde Oliveto- $3^{\mathrm{a}}$ planta \\ 31002 Pamplona \\ Tfno.- 948429308 \\ e-mail: evamarialasa@mixmail.com
}




\section{INTRODUCCIÓN}

El tratamiento de la alergia respiratoria, rinoconjuntivitis y asma bronquial incluye cuatro pilares: la educación de paciente, las medidas de desalergenización (evitación del alergeno), el tratamiento sintomático farmacológico y la inmunoterapia específica (ITE).

La ITE consiste en la administración de dosis progresivamente crecientes del alergeno al que el paciente se halla sensibilizado, con el objetivo de alcanzar una hiposensibilización y reducir o curar los síntomas que se suceden a la exposición de dicho alergeno.

Desde los primeros ensayos con inmunoterapia en $1911^{1}$ hasta nuestros días, son numerosos los estudios que demuestran su eficacia. Tal es así, que en 1998 la OMS lo avala como tratamiento de las enfermedades alérgicas ${ }^{2}$. Además, se ha demostrado que por el momento, es el único tratamiento capaz de modificar la historia natural de la enfermedad, por ejemplo la instauración de asma en pacientes con rini- tis ${ }^{3}$, y de prevenir el desarrollo de nuevas sensibilizaciones ${ }^{4,5}$. Es también por estos motivos, y no únicamente por el alivio sintomático de la enfermedad, por lo que el tratamiento inmunoterápico debería ser valorado en el paciente alérgico. La decisión de inicio de inmunoterapia se hará en base al cumplimiento de unos requisitos previos (Tabla 1) y a las indicaciones (Tabla 2) y contraindicaciones (Tabla 3 ) en el caso particular de cada paciente.

La vía clásica de administración de inmunoterapia es la vía subcutánea, cuya eficacia y seguridad ha sido demostrada en numerosos estudios, plantea varios inconvenientes como la aparición de efectos adversos, la necesidad de inyecciones en cada aplicación y el desplazamiento del paciente a un centro sanitario para su administración. Por todo esto y por la necesidad de nuevas alternativas en el terreno de la inmunoterapia se han buscado nuevas vías de administración que pudieran minimizar los efectos adversos y fuesen así mismo más cómodas: las llamadas inmunoterapias locales o no inyecta-

Tabla 1. Requisitos previos al inicio de inmunoterapia

1. Existencia demostrada de una enfermedad mediada por IgE: pruebas cutáneas positivas y/o IgE específica en suero.

2. Evidencia de que la sensibilización específica está implicada en los síntomas mediante prueba de provocación con el/los alergenos relevantes si fuera necesario.

3. Caracterización de otras factores desencadenantes que pueden estar implicados en los síntomas

4. Gravedad y duración de los síntomas:

- Síntomas subjetivos.

- Parámetros objetivos (como absentismo escolar o laboral).

- Parámetros de función pulmonar: debe excluirse a pacientes con asma grave.

- Monitorización de la función pulmonar por pico-flujo.

5. Respuesta de los síntomas al tratamiento no inmunológicos: - Respuesta a la evitación del alergeno.

- Respuesta a la farmacoterapia.

6. Disponibilidad de vacunas estandarizadas o de alta calidad.

7. Contraindicaciones

- Tratamiento con b-Bloqueantes

- Otras enfermedades inmunológicas.

- Inadecuado cumplimiento por parte del paciente.

8. Factores sociológicos:

- Coste.

- Problemas laborales (ocupación, etc).

- Deterioro en la calidad de vida a pesar de un tratamiento farmacológico adecuado.

9. Evidencia objetiva de la eficacia de la ITE para el paciente seleccionado (disponiblidad de ensayos clínicos controlados) 
Tabla 2. Indicaciones de la inmunoterapia específica.

\section{RINITIS ALÉRGICA}

- Cuando el tratamiento farmacológico es insuficiente para controlar los síntomas.

- Cuando los pacientes no desean realizar un tratamiento farmacológico.

- Cuando los pacientes no desean recibir un tratamiento farmacológico a largo plazo.

- Cuando el tratamiento médico provoque efectos adversos no deseados.

- Cuando se desea modificar el curso natural de la enfermedad alérgica con progresión hacia asma bronquial.

\section{ASMA ALÉRGICO}

Cuando los pacientes no presenten una forma grave de enfermedad: los valores del volumen espiratorio máximo expulsado en 1 seg (VEMS) deberían ser mayores del $70 \%$ del valor esperado después de un tratamiento farmacológico adecuado.

- Cuando los síntomas no se controlen de una manera adecuada mediante la evitación del alergeno y el tratamiento farmacológico.

- Cuando los pacientes presenten tanto síntomas nasales como bronquiales.

- Cuando los pacientes no desean someterse a un tratamiento farmacológico a largo plazo.

- Cuando el tratamiento farmacológico provoque efectos adversos no deseados.

Tabla 3. Contraindicaciones.

\section{ABSOLUTAS}

- Enfermedades inmunopatológicas e inmunodeficiencias graves.

- Enfermedades malignas.

- Trastornos psicológicos intensos.

\section{RELATIVAS}

- Tratamiento con $\beta$-Bloqueantes.

- Mal cumplimiento.

- Asma grave mal controlado.

- Enfermedades cardiovasculares importantes que aumenten el riesgo de efectos adversos tras el uso de adrenalina.

- Niños menores de 5 años.

- Embarazo. bles. Hasta la fecha se han desarrollado 4 vías de administración alternativas a la inmunoterapia subcutánea: la vía bronquial, nasal, oral y sublingual. De estas, es la inmunoterapia sublingual la que mayor relevancia tiene en el momento actual.

\section{TIPOS DE INMUNOTERAPIA LOCAL}

\section{Inmunoterapia bronquial}

La inmunoterapia bronquial (ITB) se propuso en $1951,{ }^{6}$ pero no se demostró su eficacia clínica y el balance riesgo/beneficio se consideró desfavorable por presentar algunos pacientes broncoespasmo tras su administración. Por este motivo, su uso ha sido abandonado.

\section{Inmunoterapia nasal}

La inmunoterapia nasal (ITIN) fue propuesta en los años 70 bajo la hipótesis de que, mediante la estimulación repetida de la mucosa nasal con bajas dosis de alergeno, se podría llegar a obtener una disminución en la reactividad de la misma. ${ }^{7}$ Los extractos que se han usado para este tipo de inmunoterapia han sido los acuosos, altamente eficaces pero con el inconveniente de que producen con frecuencia rinitis tras su administración, y los alergoides (alergenos modificados químicamente), sin tantos efectos secundarios pero menos potentes. Los extractos preparados como polvo seco (40-50 $\mathrm{mm}$ ), no provocan síntomas nasales y permiten una difusión 
uniforme por la mucosa nasal. Aunque aún no existen datos consistentes que demuestren cambios inmunológicos sistémicos tras su utilización, varios estudios han demostrado la eficacia clínica de este tipo de inmunoterapia en el tratamiento de la rinitis, tanto en reducción de síntomas como en reducción en la necesidad de medicación ${ }^{8-15}$. Los estudios han sido realizados principalmente con distintos pólenes en población adulta, por lo que el uso de la inmunoterapia nasal en la rinitis por ácaros y en edad pediátrica no está todavía suficientemente avalada. Además, el único estudio de seguimiento publicado hasta la fecha sugiere que la mejoría clínica no se mantiene tras la finalización de tratamiento $^{16}$. Por todo ello, y por el más fácil manejo de la inmunoterapia sublingual respecto a la nasal (con la que son frecuentes los errores técnicos de administración), la vía de administración intranasal está actualmente en desuso.

\section{Inmunoterapia oral}

La inmunoterapia oral (ITO), en la que el alergeno es inmediatamente deglutido, ha constituido el punto de partida de la inmunoterapia sublingual. El desarrollo de esta vía de inmunoterapia partió de la base de que, al ser el tracto digestivo rico en tejido inmune asociado a mucosas (TLAM), era esperable una eficaz intervención de las células presentadoras de antígeno en este lugar. Los estudios comenza- ron en los años $80^{17,18}$, pero desde su inicio los resultados de eficacia obtenidos fueron negativos o como mínimo controvertidos Se consiguieron demostrar cambios inmunológicos tanto locales como sistémi$\cos ^{19,20}$, pero la eficacia clínica era parcial y únicamente con altas dosis de alergeno. Los efectos secundarios, dosis dependientes, eran principalmente gastrointestinales $\mathrm{y}$, aunque no se registraron reacciones adversas graves, no se consiguieron minimizar con el uso de cápsulas. Por todo esto, el uso clínico de esta vía de inmunoterapia se suspendió a principio de los años 90 .

\section{Inmunoterapia sublingual}

La inmunoterapia sublingual (ITSL) es aquella en la que el alergeno es mantenido debajo de la lengua 1 ó 2 minutos y después es ingerido o escupido. Es la inmunoterapia local más ampliamente extendida en la actualidad en Europa y por ello le prestaremos una especial atención en esta revisión.

La inmunoterapia sublingual se desarrolló buscando una vía rápida de administración para evitar así la degradación gas trointestinal del extracto $y$, aunque se ha demostrado que las cantidades de extracto alergénico absorbidas no son relevantes, su eficacia la avalan un gran número de ensayos clínicos.

Como se ha comentado previamente, en este tipo de inmunoterapia, el extracto

Tabla 4. Fracaso de la inmunoterapia.

\section{- Falta de aplicación de las medidas de control ambiental por parte del paciente.}

- Inadecuada selección de los alergenos incluidos en la IT, bien por un diagnóstico incorrecto, por la falta de un alergeno relevante o por un número excesivo de alergenos en pacientes polisensibilizados.

- Desarrollo de sensibilización a nuevos alergenos durante el curso de la IT.

- Potencia insuficiente del extracto.

- Error en la aplicación de la dosis.

- Abandono por parte del paciente ante la ausencia de mejoría clínica en períodos iniciales del tratamiento.

- Discordancia entre el período de administración y la visita al alergólogo como consecuencia de las listas de espera, sobre todo para pautas preestacionales.

- Refractariedad al tratamiento. 
se coloca, como su propio nombre indica, debajo de la lengua. Allí es mantenido durante 1 ó 2 minutos y posteriormente es deglutido (modalidad deglutida) o escupido (modalidad escupida). La ITSL se administra generalmente en 2 fases: fase de inicio y fase de mantenimiento. En la primera fase se administra el extracto a dosis progresivamente crecientes hasta alcanzar aquella que se ha propuesto como dosis terapéutica eficaz y, en la segunda fase, la dosis máxima alcanzada en la fase de inicio se administra entre 2 y 3 veces por semana durante un período de 3 a 5 años. La ITSL puede ser administrada de forma pre-estacional (previa al período de mayor nivel ambiental de alergeno), pre-coestacional (en la fase previa y concomitantemente a la exposición alergénica) o de forma continuada. Las dos primeras formas de administración se emplean en el tratamiento de la patología alérgica por sensibilización a pólenes, y la forma continuada cuando son perennes los alergenos causantes de la sensibilización.

\section{Mecanismos de acción}

Los mecanismos de acción de la inmunoterapia subcutánea han sido ampliamente estudiados, conociéndose varias de las modificaciones inmunológicas que se suceden a este tratamiento. Sin embargo, son poco conocidos los mecanismos de acción de la ITSL, ya que la mayoría de los trabajos realizados con este tipo de inmunoterapia han sido clínicos.

Se han descrito diversas variaciones en los niveles séricos de IgE e IgG4, total y específicas, pero estos cambios no son constantes ni reproducibles. Del mismo modo, hay estudios que encuentran una descenso significativo tanto en el número de neutrófilos y eosinófilos en el cepillado nasal como en la expresión de la molécula de adhesión ICAM-1 en las células epiteliales nasales de los pacientes tratados con ITSL, pero estos resultados han sido escasamente contrastados.

\section{Eficacia}

La eficacia de la ITSL se ha demostrado tanto en el tratamiento de la rinitis como en el asma bronquial y tanto en niños como en adultos. Los estudios de eficacia se realizan en base a la mejoría de síntomas, necesidad de medicación y, en algún caso, en base a la respuesta biológica a pruebas de provocación "in vivo". El primer estudio acerca de la eficacia de la ITSL realizado mediante un ensayo clínico doble ciego controlado con placebo, se publicó en $1986^{21}$ y, hasta la fecha, han sido 23 los ensayos de este tipo publicados. Recientemente Malling y $\mathrm{col}^{22}$ publican una revisión de todos ellos concluyendo que en el $26 \%$ de los estudios se demuestra que la ITSL es eficaz (reducción mayor del $30 \%$ tanto en síntomas como en necesidad de tratamiento sintomático), es posiblemente eficaz en el 35\% (reducción mayor del 30\% únicamente en síntomas o en la necesidad de tratamiento farmacológico) e ineficaz en el 39\% (hallazgos discordantes en cuanto a los síntomas y la necesidad de tratamiento de rescate).

A la vista de los estudios publicados, en 1998 la OMS concluyó que la ITSL y la ITIN son alternativas válidas a la ITSC y que su uso en adultos está justificado. Al poco tiempo, estas conclusiones fueron confirmadas por la Academia Europea de Alergología e Inmunología Clínica y en el año 2001, el documento ARIA (Rinitis Alérgica y su Impacto en Asma) extendió el uso de la ITSL a la edad pediátrica.

Los extractos alergénicos utilizados han sido la mayoría extractos sin modificar, y en algún caso alergoides. Los alergenos testados en estos estudios de eficacia han sido ácaros (D. pteronyssinus y $D$. pharinae) y diversos pólenes (gramíneas parietaria, olivo, ciprés, abedul, aliso y avellano), pero aún no hay estudios doble ciego controlados con placebo con extracto del hongo Alternaria ni de epitelios de animales.

Por otro lado, a diferencia de la ITSC que ha demostrado eficacia a largo plazo en la modificación de la historia natural de la enfermedad y en la prevención de nuevas sensibilizaciones en varios estudios ${ }^{3,23}$ sólo existe un estudio prospectivo que demuestra el mantenimiento de la eficacia de la ITSL a los 5 años de la finalización de tratamiento ${ }^{24}$. Son por tanto necesarios un 
mayor número de ensayos clínicos que estudien este aspecto de la ITSL.

Las causas más frecuentes de ineficacia de inmunoterapia se detallan en la tabla 4.

\section{Seguridad}

Debido a que uno de los objetivos principales de la ITSL era la de ofrecer una alternativa más segura a la ITSC, son numerosos los estudios que han centrado su atención en este aspecto.

En la mayoría de los estudios no se encuentran diferencias significativas en los efectos adversos recogidos en los grupos placebo respecto a los recogidos en los que reciben el tratamiento activo. En cualquier caso, los efectos secundarios que se describen son siempre leves y similares en niños y en adultos. En un reciente estudio sobre farmacovigilancia ${ }^{25}$, se concluye que los efectos adversos son poco frecuentes, y que el $90 \%$ de ellos son leves y no requieren tratamiento médico para su resolución. El $50 \%$ de estos efectos adversos corresponden a prurito oral, seguido en frecuencia por rinorrea y estreñimiento, siendo la urticaria y el asma infrecuentes. Las molestias gastrointestinales también han sido descri- tas, son más frecuentes en niños y son consideradas reacciones locales.

A la vista de los estudios publicados, el documento ARIA sugiere que la ITSL puede administrarse de manera segura tanto en niños como en adultos.

\section{Dosis óptima}

No existen estudios en los que se determine la dosis óptima de alergeno para el uso de ITSL, y los diferentes métodos de estandarización alergénica usados por los distintos laboratorios impiden la comparación entre los distintos extractos. Esto explica que encontremos un amplio rango en la dosis óptima utilizada en los distintos estudios. Basado en la literatura disponible, la dosis efectiva de alergeno para ITSL se sitúa entre 3-5 y 375 veces la dosis de ITSC $^{26}$. Este intervalo es amplio y en la actualidad no existen pruebas de que una dosis sea mejor que otra en cuanto a eficacia. En particular, se desconoce el comportamiento a dosis superiores a 375 veces la ITSC. Se postula que dosis bajas darían lugar a una respuesta ineficaz y dosis muy elevadas a un incremento en los efectos adversos, pero no se ha encontrado evi-

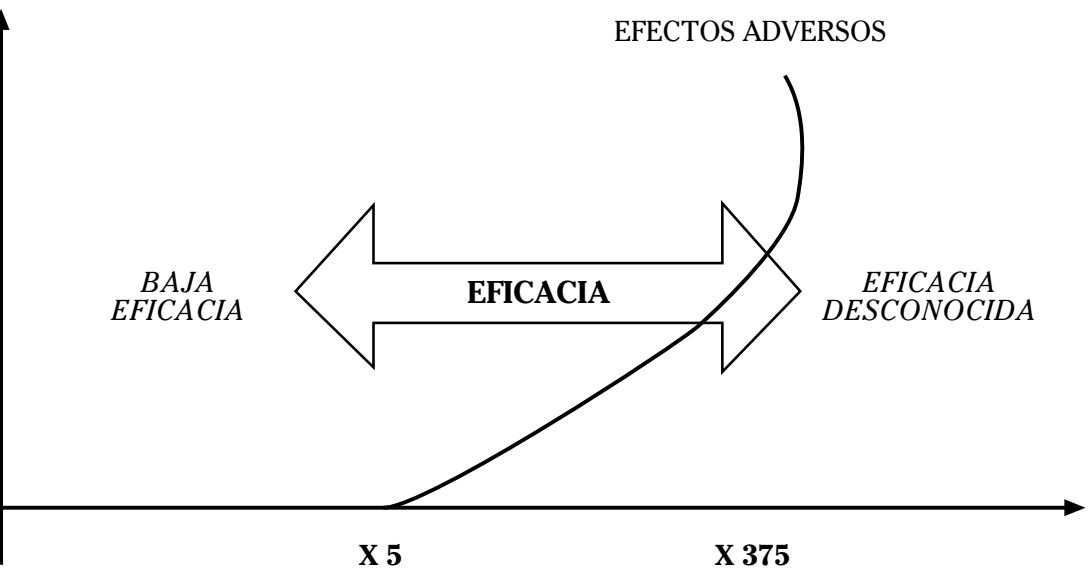

Figura 1. Dosis-respuesta relacionada con ITSL. Se ha demostrado eficacia para rango de dosis entre 20 y 375 veces la dosis de ITSC. Los efectos adversos gastrointestinales aumentan a medida que se aumenta la dosis. 
dencia de que la eficacia sea dosis dependiente (Fig. 1). Por tanto, ésta es otra cuestión todavía sin resolver en el tratamiento con ITSL.

\section{Duración de la inmunoterapia}

No existe un período de tiempo definido para mantener el tratamiento, aunque suele recomendarse como tiempo óptimo de 3 a 5 años para asegurarse la obtención de los máximos beneficios. Una duración inferior a los 3 años se asocia a un mayor índice de recaídas. Antes del primer año de tratamiento ya pueden observarse los efectos positivos de la inmunoterapia.

Siempre debe valorarse la suspensión del tratamiento en los siguientes casos:

- Resultado clínico positivo: enfermo asintomático o con reducción importante de las manifestaciones clínicas durante 2 años consecutivos.

- Resultado clínico negativo: enfermo que no responde o sin beneficio tras 1-2 años de tratamiento.

- Paciente no colaborador: incumplimiento o mal seguimiento, no justificado.

- Contraindicaciones: si aparece alguna, debe evaluarse la suspensión de la inmunoterapia.

- Reacciones anafilácticas graves.

\section{Adherencia al tratamiento y coste}

En un principio, la adherencia a la ITSL podría considerarse menor que a la ITSC ya que la administración de la primera depende del propio paciente, mientras que la segunda es administrada y controlada por personal sanitario. Aún así, el abandono de la ITSC se estima entre el 10 y $34 \%^{27}$ y hasta un $50 \%$ de los pacientes no cumplen el tratamiento debido a sus efectos adversos. En este sentido la adherencia a la ITSL, dada su seguridad, sería mayor. No obstante aún son necesarios estudios específicos a este nivel.

En cuanto al coste del tratamiento, al contener la ITSL mayor cantidad de alergeno, su precio es mayor que la ITSC. Sin embargo, la ITSL no conlleva los gastos de personal sanitario que la vía subcutánea ocasiona en su administración.

\section{CONCLUSIÓN}

El tratamiento de la enfermedad alérgica se basa en la evitación alergénica, el tratamiento farmacológico sintomático y la inmunoterapia específica. La vía subcutánea es la vía clásica de administración de este tipo de tratamiento pero en los últimos años se han desarrollado otras vías alternativas de administración de la inmunoterapia: bronquial, nasal, oral y sublingual. Mientras la ITO y la ITB no se han demostrado eficaces, existen estudios que avalan la eficacia de la ITIN y la ITSL. Es ésta última la que en la actualidad tiene mayor aceptación y su uso se está generalizando en la práctica clínica diaria.

La ITSL se ha demostrado eficaz y segura en el tratamiento de la rinitis y del asma alérgico, tanto en niños como en adultos. Sin embargo, existen todavía incógnitas acerca de este tratamiento que quedan aún sin aclarar y necesitan ser avaladas mediante estudios controlados como son: el mecanismo de acción, la eficacia a largo plazo, su efecto en la evolución de la enfermedad así como en el desarrollo de nuevas sensibilizaciones, la eficacia con otros alergenos y la dosis óptima eficaz.

Por todo esto, algunos autores sugieren que la ITSL se reserve para pacientes (con rinitis y/o asma por ácaros o pólenes insuficientemente controlados con farmacoterapia tradicional) que hayan presentado reacciones sistémicas durante la ITSC o rechacen las inyecciones ${ }^{22}$. Otros autores, por el contrario, proponen que no sea utilizada como última alternativa de tratamiento inmunoterápico, sino como una opción terapéutica más ${ }^{26}$.

\section{BIBLIOGRAFÍA}

1. Noon L. Prophylactic inoculation against hay fever. Lancet 1911: i; 1572-1573.

2. Bousquet J, Lockey RF, Malling H.G. Who Position Paper. Allergen Immunotheraphy: therapeutic vaccines for allergic diseases. Allergy 1998; 53: 1-42.

3. Moller C, Dreborg S, Ferdousi HA, Halken S, Host A, YACOBSEN L et al. Pollen immunotherapy reduces the development of asthma in children with seasonal rhinoconjunctivitis (the PAT-study). J Allergy Clin Immunol 2002; 109: 251-256. 
4. Des Roches A, Paradis L, Menardo JL, Bouges S, DAURES JP, BOUSOUET J. Immunotherapy with a standardized Dermatophagoides pteronyssinus extract. VI. Specific immunotherapy prevents the onset of new sensitizations in children. J Allergy Clin Immunol 1997; 99: 450-453.

5. Pajno GB, Barberio G. De luca F, Morabito L, PARMIANI S. Prevention of new sensitizations in asthmatic children monosensitized to house dust mite by specific immunotherapy. A six-year follow-up study. Clin Exp Allergy 2001; 31: 1392-1397.

6. HerXeIMER H. Bronchial hypersensitization and hyposensitization in man. Int Arch Allergy Appl Immunol 1951; 40: 40-57.

7. TAYlor G, Shivalkar E. Changes in nasa airways resistance on antigenic challenge in allergic rhinitis. Clin Allergy 1971; 1: 63-73.

8. NicKelsen JA, Goldstein S, Mueller U, Wypych J, REISMAN RE, ARBESMAN CE Local intranasal immunotherpy for ragweed allergic rhinitis: clinical response. J Allergy Clin Immunol 1981; 68: 33-40.

9. Passalacqua G, Albano M, Ruffoni S, Pronzato C, RICCIO AM, Di BERARDINo L et al. Local nasal immunotherapy to parietaria: evidence of reduction of allergic inflammation. Am J Respir Crit Care Med 1995; 152: 461-466

10. Andri L, Senna G, Betteli C, Givanni S, Andri G DIMITRI $\mathrm{G}$ et al. Local nasal immunotherapy with extract in powder form is effective and safe in grass pollen rhinitis: a double blind study on 32 patients. J Allergy Clin Immuno 1996; 97: 34-41.

11. Bardare M, Zaini C, Novembre E, Vierucci A Local nasal immunotherapy with a powder extract for grass pollen rhinitis in pediatric ages: a controlled study. J Investig Allergol Clin Immunol 1996; 97: 34-41.

12. Bertoni M, Cosmi F, Bianchi I, Di Berardino L. Clinical efficacy and tolerability of a steady dosage schedule of local nasal immunotherapy: results of preseasonal treatment in grasss pollen rhinitis. Ann Allergy Asthma Immunol 1999; 82: 47-51.

13. Motta G, Passali D, De Vicentis I, Ottaviani A MAURIZI M, SARTORIS A et al. A multicenter tria of specific local nasal immunotherapy. Laryngoscope 2000; 110: 132-139.

14. Pocobelli D, Del Bono A, Venuti L, Falagiani P, VENUTI A. Nasal immunotherapy at constant dosage: a double blind placebo controlled study in grass allergic rhinoconjunctivitis. Investing Allergol Clin Immunol 2001: 11: 7988
15. Marcucci F, Sensi LG, Caffarelli C, Cavagni G, BERNARDINI R, TIRI A et al. Low-dose local nasal immunotherpy in children with prennia allergic rhinitis due to Dermatophagoides. Allergy 2002; 57: 23-28.

16. Passalacqua G, Albano M, Pronzato C, Riccio A, Falagiani P, CANONica GW. Nasa immunotherapy to Parietaria: long term follow up of a double blind study. Clin Exp Allergy 1997: 27: 904-908.

17. Rebien W, Wahn U, Puttonen E, MaAsch HG. Comparative study of immunological and clinical efficacy of oral and subcutaneous hyposensitization. Allergologie 1980; 3: 101109

18. TAUDORF E, WEEKE B. Orally administered grass pollen. Allergy 1983: 38: 561-564.

19. Giovane A, Bardare M, Passalacoua G, Ruffoni S, Scordamaglia A, GHEzzi E et al. A three year double blind placebo-contolled study with specific oral immunotherapy to Dermatophagoides: evidence of safety and efficacy in pediatric patients. Clin Exp Allergy 1994; 24: 53-59.

20. TAudorf E, Laursen L, LanNer A, BJorksten B Dreborg S, WeEKe B. Specific, IgE, IgG and IgA antibody response to oral immunotherapy in birch pollenosis. J Allergy Clin Immuno 1989; 83: 589-594.

21. ScADDing K, BRostoff J. Low dose sublingua therapy in patients with allergic rhinitis due to dust mite. Clin Allergy 1986; 16: 483-491.

22. MALLING HJ. Is sublingual immunotherapy clinically effective? Curr Opin Allergy Clin Immunol 2002; 2: 523-531.

23. Passalacqua G, Canonica GW. Long lasting efficacy of specific immunotherapy. Allergy 2002; 57: 275-276.

24. Di Rienzo V, Marcucci F, Puccinelli P, Parmian $\mathrm{S}$, FRATI F, SENSI L et al. Long lasting effect of sublingual immunotherapy in children with asthma due to house dust mites: a ten-year prospective study. Clin Exp Allergy 2003; 33 206-210

25. Almangro E, Assensio O, Bartolomé JM Bosoue M, De la Hoz B, Dolz I et al. Estudio multicéntrico de farmacovigilancia de inmunoterapia sublingual en pacientes alérgicos. Allergol immunopathol 1995; 23. 153-159.

26. Canonica GW, Passalacqua G. Noninjection routes for immunotherapy. J Allergy Clin Immunol 2003; 111: 437-448.

27. LOWER T, HENSY J, MANDIK L, JANOSKY J, FRIDAY GA JR. Compliance with allergen immunotherapy. Ann Allergy 1993; 70: 480-482. 